\title{
OPENNESS OF VECTOR MEASURES AND THEIR INTEGRAL MAPS
}

\author{
ANDRZEJ SPAKOWSKI \\ (Received 30 June 1986; revised 2 November 1987) \\ Communicated by J. F. Price
}

\begin{abstract}
We prove that finite dimensional nonatomic vector measures and their integral maps are open maps. These results can be found in the literature, but unfortunately the proofs presented there are not complete.

1980 Mathematics subject classification (Amer. Math. Soc.): primary 28 B 05; secondary 54 C 10.
\end{abstract}

\section{Introduction}

The problem of openness of vector measures and their integral maps has been treated by Anantharaman and Garg [2]. Earlier Karafiat [12] considered a continuity property of the map inverse to a vector measure which gives, in particular, the openness of the measure. Samet [16] rediscovered the problem of openness, but his proof of the openness of finite dimensional nonatomic measures contains a gap (see [16, page 472], the assertion of the openness of $\mu(\Omega(S, \varepsilon))$ ). Recently Professor Samet informed me that he could fill the gap. Incomplete also are some proofs in [2] and [12] (see the last section of this paper).

Our aim is to prove that $\mathbf{R}^{p}$ valued nonatomic measures and their integral maps are open maps. Our arguments follow, in part, [2], [12] and [15], but we include some of them for the sake of completeness.

(C) 1988 Australian Mathematical Society $0263-6115 / 88 \$ A 2.00+0.00$ 
In this section we introduce the notation and recall some preliminary results. In Section 2 we state a few characterizations of open maps. Section 3 contains some results on semiconvex measures. Section 4 contains some rather general results on points of openness of vector measures and affine maps. In Section 5 we present a decomposition lemma due to Liapunov [15] and Karafiat [12]. The main results are presented in Section 6. Some applications of openness of vector measures and their integral maps are contained in the next section. The last section contains some remarks.

Throughout this paper $S$ is a nonempty set, $\mathscr{A}$ is a $\sigma$-algebra of subsets of $S$ and $m$ is a vector measure on $\mathscr{A}$ with values in a complete metrizable locally convex space $Y$. It is known that there exists a finite positive measure $\mu$ on $\mathscr{A}$ equivalent to $m[4 ; 13$, page 21$] ; \mu$ is called a control measure of $m$. Let $P_{0}$ be the set of all characteristic functions of sets in $\mathscr{A}$ and $P$ the set of all $\mathscr{A}$-measurable $[0,1]$-valued functions in $S$. We will identify $P_{0}$ with $\mathscr{A}$. Note that $P_{0} \subset P \subset L^{\infty}$, where $L^{\infty}=L^{\infty}(\mu)$. Let $T_{m}$ be the integral map of $m$ from $P$ into $Y$ defined by $T_{m}(x)=\int x d m$ for $x \in P$, where the integral is in the sense of [13, page 26]. On $P_{0}$ we will identify $T_{m}$ with $m$.

The sets $P_{0}$ and $P$ are considered under the topology $w^{*}$, the weak-star topology of $L^{\infty}$. On $P_{0}$ this topology is identical with the Fréchet-Nikodym topology given by the metric $d(A, B)=\mu(A \triangle B)$, where $A \triangle B$ denotes the symmetric difference of $A$ and $B$. On $P$ the topology $w^{*}$ is identical with the weak topology of $L^{1}=L^{1}(\mu)$. The integral map $T_{m}$ is continuous as a map from $P$ into $(Y, \sigma)$, where $\sigma$ is the weak topology of $Y$ (see [13, page 68]). This follows easily by applying the Radon-Nikodym theorem. The measure $m$ is continuous as a map from $P_{0}$ into $Y$.

Let $K$ denote the range of the integral map $T_{m}$. It is known that $K$ is the closed convex hull of the range of $m[13$, page 76].

\section{Open maps}

Let $X$ and $Y$ be topological spaces and let $T: X \rightarrow Y$ be surjective. We say that $T$ is open at $x \in X$ if for each neighbourhood $U$ of $x$ the image $T(U)$ is a neighbourhood of $T(x)$. We say that $T$ is open if for each open set $U$ in $X$ the image $T(U)$ is open in $Y$. Clearly, $T$ is open if and only if $T$ is open at each point of $X$.

Denote by $2^{X}$ the family of all subsets of $X$ and let $F$ be a multifunction from $Y$ to $X$, that is, $F: Y \rightarrow 2^{X}$. We say that $F$ is lower semicontinuous at $y_{0} \in Y$ if for each open set $U$ in $X$ satisfying $F\left(y_{0}\right) \cap U \neq \varnothing$ there exists a neighbourhood $V$ of $y_{0}$ such that $F(y) \cap U \neq \varnothing$ for all $y \in V$. Given a map 
$T: X \rightarrow Y$ we denote by $T^{-1}$ the multifunction from $Y$ to $X$ defined by the formula $T^{-1}(y)=\{x \in X: T(x)=y\}$.

Let $A_{\alpha}$ be a net of subsets of $X$. We define $L i A_{\alpha}$ as the set of all $x \in X$ such that for each neighbourhood $U$ of $x$ there exists $\alpha_{0}$ such that $A_{\alpha} \cap U \neq \varnothing$ for all $\alpha>\alpha_{0}$, and $L_{s} A_{\alpha}$ as the set of all $x \in X$ such that for each neighbourhood $U$ of $x$ and each $\alpha$ there exists $\beta>\alpha$ with $A_{\beta} \cap U \neq \varnothing$ (see [14, Section 29]).

It is easy to prove the following lemma.

LEMMA 2.1. Let $X$ and $Y$ be topological spaces, $T: X \rightarrow Y$ be surjective and $y_{0} \in Y$. Consider the following conditions.

(a) $T$ is open at each $x \in T^{-1}\left(y_{0}\right)$.

(b) The multifunction $T^{-1}$ is lower semicontinuous at $y_{0}$.

(c) $T^{-1}\left(y_{0}\right) \subset L i T^{-1}\left(y_{\alpha}\right)$ for each net $\left(y_{\alpha}\right)$ in $Y$ converging to $y_{0}$.

(d) $T^{-1}\left(y_{0}\right) \subset L s T^{-1}\left(y_{\alpha}\right)$ for each net $\left(y_{\alpha}\right)$ in $Y$ converging to $y_{0}$.

(e) For each $x \in T^{-1}\left(y_{0}\right)$ and each net $\left(y_{\alpha}\right)$ in $Y$ converging to $y_{0}$ there exists a net $\left(x_{\alpha}\right)$ in $X$ converging to $x$ such that $x_{\alpha} \in T^{-1}\left(y_{\alpha}\right)$ for all $\alpha$.

(f) $T^{-1}\left(y_{0}\right)=L s T^{-1}\left(y_{\alpha}\right)$ for each net $\left(y_{\alpha}\right)$ in $Y$ converging to $y_{0}$.

The conditions (a), (b), (c) and (d) are equivalent. Condition (e) implies (d). If $X$ and $Y$ are first countable then (d) implies (e) (in this case we replace nets in these conditions by sequences). If $T$ is continuous and $Y$ is a Hausdorff space, then (f) is equivalent to (d). In case $Y$ is metrizable we may replace in the above nets by sequences.

Note that condition (f) in Lemma 2.1 is due to Hájek [9]. The equivalence of conditions (a), (c) and (f) generalizes some results of Sikorski [17, page 16] and Hájek [9, Proposition 1].

\section{Semiconvex measures}

A measure $m: \mathscr{A} \rightarrow Y$ is called semiconvex if for each $A \in \mathscr{A}$ there exists $B \in \mathscr{A}$ such that $B \subset A$ and $m(B)=m(A) / 2$ (see [10]). As easily seen, a semiconvex measure is nonatomic. In view of Liapunov's theorem [15, Theorem 1] the converse holds in case $Y=\mathbf{R}^{p}$. We shall need the following known lemma [3].

LEMMA 3.1. If $m: \mathscr{A} \rightarrow Y$ is semiconvex, then

(1) $\operatorname{ext} T_{m}^{-1}(y)=m^{-1}(y)$ for each $y \in m\left(P_{0}\right)$,

(2) $T_{m}(P)=m\left(P_{0}\right)$. 
Indeed, by [3, Theorem 2.2] we have ext $T_{m}^{-1}(y) \subset P_{0}$ for each $y \in T_{m}(P)$. Since ext $P=P_{0}$, this yields (1). In view of the Krein-Milman theorem, (1) implies (2).

The next lemma implies that, in case $m$ is semiconvex and finite dimensional, the openness of $T_{m}$ and that of $m$ are equivalent. The second part of our proof follows an argument of [2, proof of Proposition 2.6].

LEMMA 3.2. Let $m: \mathscr{A} \rightarrow Y$ be a semiconvex measure and let $K$, the common range of $m$ and $T_{m}$, be equipped with the weak topology $\sigma$. Then, for every $y_{0} \in K$, the following conditions are equivalent:

(a) $m$ is open at each $x \in m^{-1}\left(y_{0}\right)$;

(b) $T_{m}$ is open at each $x \in T_{m}^{-1}\left(y_{0}\right)$.

Proof. Assume (a) and let $\left(y_{\alpha}\right)$ be a net in $K$ converging to $y_{0}$. Then, in view of Lemma 2.1 we have $m^{-1}\left(y_{0}\right) \subset L i m^{-1}\left(y_{\alpha}\right)$. Hence, by Lemma 3.1(1), $\operatorname{ext} T_{m}^{-1}\left(y_{0}\right) \subset L i T_{m}^{-1}\left(y_{\alpha}\right)$. This yields in view of the Krein-Milman theorem that

$$
T_{m}^{-1}\left(y_{0}\right) \subset \operatorname{col} L i T_{m}^{-1}\left(y_{\alpha}\right)
$$

Since the limes inferior of a net of closed and convex sets is closed and convex, it follows that

$$
T_{m}^{-1}\left(y_{0}\right) \subset L i T_{m}^{-1}\left(y_{\alpha}\right) .
$$

In view of Lemma 2.1, this shows that (b) holds.

Assume (b) and let $\left(y_{\alpha}\right)$ be a net in $K$ converging to $y_{0}$. In view of Lemms 2.1(f), we have $T_{m}^{-1}\left(y_{0}\right)=L s T_{m}^{-1}\left(y_{\alpha}\right)$. Hence, by a result of Jerison [10], Theorem 2]

$$
\operatorname{ext} T_{m}^{-1}\left(y_{0}\right) \subset L s \operatorname{ext} T_{m}^{-1}\left(y_{\alpha}\right)
$$

or, equivalently, $m^{-1}\left(y_{0}\right) \subset L s m^{-1}\left(y_{\alpha}\right)$ (Lemma 3.1(1)). In view of Lemma 2.1 , this shows that (a) holds.

\section{Points of openness}

In this section we prove that $m$ is open at $x \in P_{0}$ whenever $m(x)$ is an extreme point of $K$, and that $T_{m}$ is open at $x \in P$ whenever $T_{m}(x)$ is an interior point of $K$.

LEMMA 4.1. If $x \in P_{0}$ and $m(x) \in \operatorname{ext} K$, then $m$ is open at $x$ (even when $K$ is considered under the weak topology $\sigma$ ).

ProOF. In view of Lemma 2.1, it is enough to find for every net $\left(y_{\alpha}\right)$ in $(K, \sigma)$ converging to $m(x)$, and every net $\left(x_{\alpha}\right)$ with $x_{\alpha} \in m^{-1}\left(y_{\alpha}\right)$, a subset $\left(x_{\beta}\right)$ of 
$\left(x_{\alpha}\right)$ convergent to $x$. Let $\left(x_{\alpha}\right)$ and $\left(y_{\alpha}\right)$ be such as above. Then $\left(P, w^{*}\right)$ is a compact space. Thus there exists a subnet $\left(x_{\beta}\right)$ of $\left(x_{\alpha}\right) w^{*}$-convergent to some $x_{0} \in P$. Since $T_{m}$ is continuous $\left(T_{m}\left(x_{\beta}\right)\right)$ converges to $T_{m}\left(x_{0}\right)=m(x)$. Hence, by $\left[13\right.$, Theorem VI.1.1], $x_{0}=x$.

Proposition 4.2. Let $X$ and $Y$ be topological linear spaces, let $C$ be a bounded convex subset of $X$ and let $T: C \rightarrow Y$ be an affine map. Then $T$ is open at each $x \in T^{-1}\left(y_{0}\right)$ whenever $y_{0}$ is an interior point of $T(C)$.

Proof. Fix a neighbourhood $V$ of zero in $X$. We first show that there exists a neighbourhood $W$ of zero in $Y$ such that

$$
T^{-1}\left(y_{0}\right) \subset T^{-1}(y)+V,
$$

whenever $y \in T(C)$ and $y-y_{0} \in W$. By assumption, there exists $\lambda>0$ with $\lambda(C-C) \subset V$. We assume that $\lambda \leq 1$. Choose a neighbourhood $W$ of zero in $Y$ with

$$
y_{0}+\frac{1}{\lambda} W \subset T(C)
$$

Fix $y_{1} \in T(C)$ and $y=(1-\lambda) y_{0}+\lambda y_{1}$. Let $x_{1} \in T^{-1}\left(y_{1}\right)$ and $x_{0} \in T^{-1}\left(y_{0}\right)$ be arbitrary. Then

$$
(1-\lambda) x_{0}+\lambda x_{1} \in T^{-1}(y)
$$

whence $x_{0} \in T^{-1}(y)+V$. In view of Lemma 2.1 it is enough to show that the multifunction $T^{-1}$ is lower semicontinuous at $y_{0}$. Let $U$ be a relatively open subset of $C$ with $T^{-1}\left(y_{0}\right) \cap U \neq \varnothing$. Fix $\bar{x} \in T^{-1}\left(y_{0}\right) \cap U$ and let $V$ be a neighbourhood of zero in $X$ such that for every $x \in C$ with $x-\bar{x} \in V$ we have $x \in U$. Taking $W$ as above, we get $T^{-1}(y) \cap U \neq \varnothing$, whenever $y \in C$ and $y-y_{0} \in W$.

COROLlaRY 4.3. Let $m: \mathscr{A} \rightarrow Y$ be a semiconvex measure and let $y_{0}$ be an interior point of $(K, \sigma)$. Then $m$ is open at each point $x \in m^{-1}\left(y_{0}\right)$.

Indeed, in view of Proposition 4.2, $T_{m}$ is open at each point $x \in T_{m}^{-1}\left(y_{0}\right)$. The assertion now follows from Lemma 3.2 .

\section{A decomposition lemma}

Properties (i)-(iv) and (v) and (vi) of the lemma below are due to Liapunov [15, page 475] and Karafiat [12, page 42], respectively. 
LEMMA 5.1. Let $m: \mathscr{A} \rightarrow \mathbf{R}^{p}$ be a nonatomic measure, $K$ its range and $H$ a supporting hyperplane of $K$. Then there exists a decomposition $m=m_{1}+m_{2}$ such that

(i) $m_{1}$ and $m_{2}$ are measures on $\mathscr{A}$ concentrated on disjoint sets $S_{1}$ and $S_{2}=S \backslash S_{1}$, respectively,

(ii) $K=K_{1}+K_{2}$, where $K_{i}$ is the range of $m_{i}, i=1,2$,

(iii) $K_{1}$ lies in the linear subspace of $\mathbf{R}^{p}$ parallel to $H$ and $\operatorname{dim} K_{1}<p$,

(iv) $K_{2}$ has only one point in $H, K_{2} \cap H=\{b\}$ say, and $b$ is an extreme point of $K_{2}$,

(v) $K \cap H=K_{1}+b$,

(vi) if $\left(y_{n}\right)$ is a sequence in $K$ convergent to $y_{0} \in K \cap H$ and $y_{n}=y_{n}^{1}+y_{n}^{2}$, where $y_{n}^{1} \in K_{1}$ and $y_{n}^{2} \in K_{2}$ for all $n$, then $\left(y_{n}^{1}\right)$ converges to $y_{0}-b$ and $\left(y_{n}^{2}\right)$ converges to $b$.

\section{Finite dimensional measures}

Now we are ready to prove that finite dimensional nonatomic measures and their integral maps are open maps.

THEOREM 6.1. Every nonatomic measure $m: P_{0} \rightarrow K$, where $K \subset \mathbf{R}^{p}$ is the range of $m$, is an open map.

ProOF. We argue by induction on $p$. If $p=1$, then $K$ is a compact interval, and so the theorem clearly follows from Lemma 4.1 and Corollary 4.3 (or from [2, Lemma 2.1]).

Let $p>1$ and suppose that $K$ spans $\mathbf{R}^{p}$. We shall prove that $m$ is open at each point $x \in P_{0}$. In view of Corollary 4.3, it is enough to consider the case where $m(x)$ is a boundary point of $K$. Then there exists a hyperplane $H$ supporting $K$ at $m(x)$. We may assume that $H \cap K \neq\{m(x)\}$ for in the contrary case $m(x) \in \operatorname{ext} K$ and the theorem follows again from Lemma 4.1. We establish the openness of $m$ at $x$ with the help of condition (e) of Lemma 2.1. Accordingly, let $\left(y_{n}\right)$ be a sequence in $K$ convergent to $y=m(x)$. Applying Lemma 5.1 and adopting its notation we see that $m_{1}(x)=y-b$ and $m_{2}(x)=b$. Moreover, $K_{1}$ spans a proper subspace of $\mathbf{R}^{p}$ and $b \in \operatorname{ext} K_{2}$. Therefore, by the induction hypothesis and Lemma 4.1, respectively, $m_{1}$ and $m_{2}$ are open at $x$. Hence there exist sequences $\left(x_{n}^{1}\right)$ and $\left(x_{n}^{2}\right)$ in $P_{0}$ convergent to $x$ such that $x_{n}^{1} \in m_{1}^{-1}\left(y_{n}^{1}\right)$ and $x_{n}^{2} \in m_{2}^{-1}\left(y_{n}^{2}\right)$ for all $n$. Let $x_{n}(t)=x_{n}^{1}(t)$ if $t \in S_{1}$ and $x_{n}(t)=x_{n}^{2}(t)$ if $t \in S_{2}$, $n=1,2, \ldots$ Then $\left(x_{n}\right)$ is a sequence in $P_{0}$ convergent to $x$ and $x_{n} \in m^{-1}\left(y_{n}\right)$ for all $n$. This completes the proof. 
Now Theorem 6.1 and Lemma 3.2 yield the following theorem.

THEOREM 6.2. If $m$ is a finite dimensional nonatomic measure, then the integral map $T_{m}: P \rightarrow K$ is an open map.

\section{Applications of openness}

Anantharaman and Garg [2, Proposition 2.3] proved that the set of extreme points of the closed convex hull of the range of a vector measure with values in $Y$ is closed provided the integral map is open. However, in the case of a semiconvex measure this result can be easily obtained by applying the following lemma (see [5, Lemma 3]): let $T$ be an open continuous map from a Hausdorff space $X$ onto a topological space $Y$; for every positive integer $n$ the set

$$
F_{n}=\left\{y \in Y: T^{-1}(y) \text { has at most } n \text { points }\right\}
$$

is closed.

THEOREM 7.1. Let $m$ be a semiconvex measure and $T_{m}$ (equivalently $m$ ) be open. Then the set of extreme points of the range of $m$ is closed.

Proof. It is known that $y \in$ ext $K$ if and only if $T_{m}^{-1}(y)=\left\{\chi_{A}\right\}$ for some $A \in \mathscr{A}$, where $K$ is the range of $T_{m}$ (see [1, Proposition 2]). However, $m$ is semiconvex and so $K$ is equal to the range of $m$. By the lemma recalled above we conclude that the set ext $K$ is closed.

COROLLARY 7.2. If $m$ is a nonatomic finite dimensional valued measure then the set of extreme points of the range of $m$ is closed.

\section{Remarks}

Let $P_{1}$ be the set of all $x \in L^{\infty}$ with values in $[-1,1] \mu$-almost everywhere, endowed as $P$ with the topology $w^{*}$. Denote by $T_{m}^{1}$ the map on $P_{1}$ defined by the formula

$$
T_{m}^{1}(x)=T_{m}((1+x) / 2) \quad \text { for } x \in P_{1},
$$

or equivalently, by the equation $T_{m}(x)=T_{m}^{1}(2 x-1)$, where $x \in P$. Clearly, $T_{m}$ and $T_{m}^{1}$ have the same range (see [8]). Hence in view of Lemma 2.1, $T_{m}$ is open if and only if $T_{m}^{\mathbf{1}}$ is open (see a problem posed in [16]). 
In [12] Karafiat applied Bolker's characterization of faces of the range of $\mathbf{R}^{p}$ valued nonatomic measure [5, Corollary 3.3]. But this characterization concerns only exposed faces. Consequently, the proof of Theorem 2 in [12] is complete only for $p \leq 2$.

Anantharaman and Garg [2, proof of Theorem 2.2] use the following assertion: the operation $(A, B) \mapsto A \cap B$ is continuous relative to the Hausdorff metric (see also [13, page 70]). However, this assertion is easily seen to be false.

\section{Acknowledgements}

I wish to express my thanks to Z. Lipecki for introducing me to the problem and for valuable suggestions. I would like to thank A. Schief from München for a correction to the formulation of Lemma 2.1. Finally, I would like to thank the referee who drew my attention to Armstrong's paper [18], where the problem of openness is examined for finitely additive measures.

\section{References}

[1] R. Anantharaman, 'On exposed points of the range of a vector measure', Proc. Snowbird sympos. on vector and operator valued measures and applications, pp. 7-22 (Academic Press, 1973).

[2] R. Anantharaman and K. M. Garg, 'Some topological properties of vector measures and their integral maps', J. Austral. Math. Soc. Ser. A 23 (1977), 453-466.

[3] R. Anantharaman and K. M. Garg, 'On the range of a vector measure', Bull. Math. Soc. Sci. Math. Roumainie 22 (1978), 115-132.

[4] R. G. Bartle, N. Dunford and J. T. Schwartz, 'Weak compactness and vector measures', Canad. J. Math. 7 (1955), 289-305.

[5] E. D. Bolker, 'A class of convex bodies', Trans. Amer. Math. Soc. 145 (1969), 323-345.

[6] W. Bula, 'On metrizability of continuous images of compact ordered spaces', Fund. Math. 123 (1984), 21-27.

[7] R. Engelking, General topology (PWN, Warszawa, 1977).

[8] P. Glicksberg, 'A controlled approach to Liapounoff convexity', J. Math. Anal. Appl. 94 (1983), 193-196.

[9] O. Hájek, 'Notes on quotient maps', Comment. Math. Univ. Carolinae 7 (1966), 319323.

[10] P. R. Halmos, 'The range of a vector measure', Bull. Amer. Math. Soc. 54 (1948), 416 421.

[11] M. Jerison, 'A property of extreme points of compact convex sets', Proc. Amer. Math. Soc. 5 (1954), 782-783.

[12] A. Karafiat, 'On the continuity of a mapping inverse to a vector measure', Comment. Math. Prace Mat. 18 (1974), 37-43.

[13] I. Kluvánek and G. Knowles, Vector measures and control systems (North-Holland, New York, 1976).

[14] K. Kuratowski, Topology I (Academic Press, New York, 1966).

[15] A. A. Liapunov, 'Sur les fonctions vecteur completement additives' (in Russian), Izv. Akad. Nauk SSSR Ser. Mat. 4 (1940), 465-478. 
[16] D. Samet, 'Vector measures are open maps', Math. Oper. Res. 9 (1984), 471-474.

[17] R. Sikorski, 'Closure homomorphisms and interior mappings', Fund. Math. 41 (1954), 12-20.

[18] T. E. Armstrong, 'Openness of finitely additive vector measures as mappings', preprint.

Institute of Mathematics

Pedagogical University

Oleska 48, 45-951 Opole

Poland 